\title{
Energy Systematics of Jet Tomography at RHIC: $\sqrt{s}=62.4$ vs $200 \mathrm{AGeV}$
}

\author{
A. Adi a and M. Gyulassy \\ Columbia University, Department of Physics, 538 West 120-th Street, New York, NY 10027
}

(Dated: November 5, 2018)

\begin{abstract}
The collision energy dependence of jet tomography is investigated within the GLV formalism. The emphasis is on estimating systematic uncertainties resulting from the interplay of energy loss fluctuations and the rapid increase of the parton transverse momentum slopes as $\sqrt{s}$ decreases from 200 to $62 \mathrm{AGeV}$.

PACS numbers: $12.38 . \mathrm{Mh} ; 24.85 .+\mathrm{p} ; 25.75 .-\mathrm{q}$
\end{abstract}

\section{INTRODUCTION}

We study the energy dependence systematics of jet tomography in nuclear collisions within the GLV formalism [1, 2] in the $\sqrt{s}=62$ to $200 \mathrm{AGeV}$ range. In general, tomography provides a map of the density of matter through the study of the angular pattern of attenuation of a calibrated source beam. Extensive high $p_{T}$ data on single and dijet azimuthal correlations and relative to the reaction plane have already been compiled at $200 \mathrm{AGeV}$ [4, 5] and used to determine opacity and density of the quark-gluon plasma (QGP) produced in central $\mathrm{Au}+\mathrm{Au}$ reactions $[3$. The interest in applying the same techniques to lower $62 \mathrm{AGeV}$ data [6] is to test experimentally the predicted $\sqrt{s}$ decrease of the QGP density and the predicted variation of the gluon/quark jet source. In this paper, we calculate the nuclear modification factor, $R_{A A}\left(p_{T}, y=0, \sqrt{s}\right)$, for central $A u-A u$ collisions at both $\sqrt{s}=62.4,200 \mathrm{AGeV}$ for neutral pions. Previous predictions for $62 \mathrm{AGeV}$ have been published by Wang [] and Vitev [8].

We concentrate here on the role of energy loss fluctuations [2, 15] on the predicted single hadron attenuation pattern in order to gain an estimate of some of the systematic theoretical errors in the jet tomographic technique. To isolate the role of fluctuations we neglect $k_{T}$ smearing, the Cronin enhancement, gluon and quark shadowing, and nonperturbative baryon dynamical contributions that strongly distort the hadron spectra below $p_{T}<4-5 \mathrm{GeV}$ 1]. Therefore, our calculations can only be compared to unidentified charged particles outside the baryon anomaly region $[9]$, i.e. $p_{T} \gtrsim 5 \mathrm{GeV}$. Even for the identified $\pi^{0}$ spectra, the calculations neglecting the rapidly rising Cronin effect with decreasing energy limits the applicability to the $p_{T}>4 \mathrm{GeV}$ region. See different estimates of those effects in Refs. 7, [8]. The in-

\footnotetext{
*Electronic address: azfar@phys.columbia.edu

${ }^{\dagger}$ Electronic address: gyulassy@phys.columbia.edu
}

termediate $p_{T}$ is also strongly influenced by the transverse Doppler shifted hydrodynamic flow of the QGP bulk matter 10] as well as possible coalescence hadronization mechanisms 11] not described by attenuated parton fragmentation.

The tomographic information about the maximum densities of the QGP attained at RHIC as a function of energy is best isolated from the $p_{T}>5 \mathrm{GeV}$ region. However, it is important to examine critically the underlying assumptions of jet tomography to assess the theoretical systematic errors in its application to nuclear reactions. We test below the influence of the shape of the energy loss fraction spectrum, $P(\epsilon, \bar{\epsilon})$ about the mean energy loss fraction, $\bar{\epsilon}$. The tomographic information is encoded in $\bar{\epsilon}$ because for Bjorken expansion it is is proportional to the produced gluon rapidity density, $d N_{g} / d y$ as well as the path length. Neglecting fluctuations as in 12], i.e., $P(\epsilon, \bar{\epsilon})=\delta(\epsilon-\bar{\epsilon})$, a fit of $R_{A A}$ provides a biased measure of $\bar{\epsilon}$ and hence $d N_{g} / d y$.

As first emphasized in [13] fluctuations about $\bar{\epsilon}$ must be considered in realistic applications because the very rapid decrease of the transverse momentum spectra of partons induces a bias to lower effective $\epsilon$. In Ref. 2] we quantified the magnitude of the fluctuation bias in the GLV approach through a renormalization factor $Z$. We showed that for $130 \mathrm{AGeV}$ conditions $Z \sim 0.5$ due to this bias, and hence the fitted $\bar{\epsilon}$ from $R_{A A}$ must be upward corrected by a factor $1 / Z \approx 2$.

At lower energies, the high $p_{T}$ slopes of the transverse momentum spectra increase more and more as $\sqrt{s}$ decreases as seen in Fig. 1. Thus the calibrated gluon and quark source beams change systematically as a function of $\sqrt{s}$. This increases the fluctuation biases and hence it is important to investigate how sensitive is the tomographic analysis to not only $\bar{\epsilon}$ but also to the shape of that distribution. 


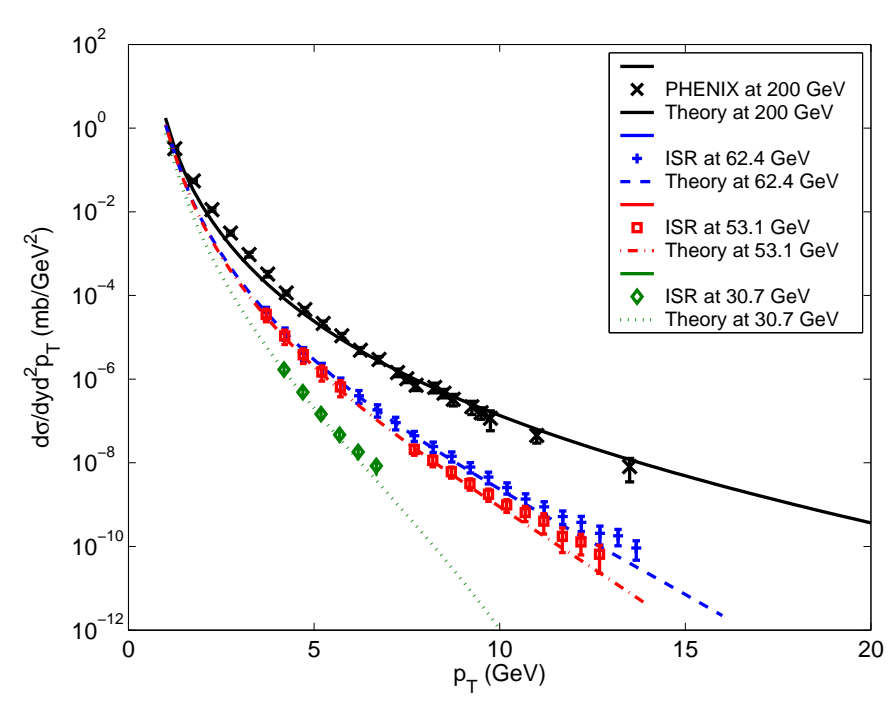

FIG. 1: Invariant cross-sections for $\pi^{0}$ production in $\mathrm{p}-\mathrm{p}$ collisions at $\sqrt{s}=30.7,53.1,62,4,200 \mathrm{GeV}$. Data is from PHENIX 4] (200 GeV) and ISR (all other energies) collaborations.

\section{CALCULATION OF SPECTRA}

The neutral pion invariant inclusive cross section in $p p$ at various values of $\sqrt{s}$ can be calculated via conventional collinear factorized pQCD,

$$
\begin{gathered}
E_{h} \frac{d \sigma_{\pi^{0}}^{p p}}{d^{3} p}=K \sum \int d x_{1} d x_{2} f_{a / A}\left(x_{1}, Q^{2}\right) f_{b / A}\left(x_{2}, Q^{2}\right) \\
\frac{d \sigma^{a b \rightarrow c d}}{d \hat{t}} \frac{D_{\pi^{0} / c}\left(z_{c}, Q^{2}\right)}{\pi z_{c}}
\end{gathered}
$$

The inclusive number distribution, $d N^{\pi^{0}} / d y d^{2} p_{T}$, in $A+A$ collisions is obtained in the absence of nuclear effects by simply multiplying Eq. 1 by the Glauber geometric (binary collision density) factor, $T_{A B}(b)$. We use standard Leading Order MRS D- parton distribution functions $f_{a}(x)$ (as in 17]), and KKP fragmentation functions $D_{h}\left(z_{c}\right)$ (as in [18]). Here, $x_{1}$ is the momentum fraction of the projectile parton in the collision while $x_{2}$ is the momentum fraction of the target parton. The $K(\sqrt{s})$ is a factor that simulates higher order effects.

Figure 1 compares the invariant pQCD cross-sections for $\pi^{0}$ production to available data. The particular values used for $K$ and $Q^{2}$ for the fits are given $K=2.0, Q^{2}=$ $p_{T}^{2}$ for $200 \mathrm{GeV}, K=2.5, Q^{2}=0.5 p_{T}^{2}$ for $62.4 \mathrm{GeV}$, $K=3.5, Q^{2}=0.5 p_{T}^{2}$ for $53.1 \mathrm{GeV}$ and $K=4.5, Q^{2}=$ $0.5 p_{T}^{2}$ for $30.7 \mathrm{GeV}$. The rapid increase of the slopes with decreasing $\sqrt{s}$ is well reproduced by pQCD. However, the normalization corrections due to NLO processes increases as $s$ decreases.

\section{FINAL STATE QUENCHING AND FLUCTUATIONS}

In a dense QCD medium the induced radiative energy loss reduces the initial transverse momentum $p_{T}^{0}$ of a produced hard parton prior to hadron fragmentation. The reduction factor $(1-\epsilon)$ depends in general on both the initial parton rapidity $y$ and transverse momentum $p_{T}^{0}$ as well as on local QGP density that varies with $s$ and impact parameter $b[1]$.

In this paper, we consider only mid-rapidity $(y=0)$ and central $b<<R$ collisions and further simplify the calculation by neglecting the slow $p_{T}$ dependence of the energy loss fraction, $\epsilon$. The parton before fragmentation has $p_{T}^{f}=p_{T}^{0}(1-\epsilon)$ in this picture. We neglect acoplanarity as well.

Fluctuation in the energy loss is taken into account via a probability function $P(\epsilon, \bar{\epsilon})$ using [2]

$$
\begin{gathered}
E_{h} \frac{d \sigma_{\pi^{0}}(\bar{\epsilon})}{d^{3} p}=K \sum \int d x_{1} d x_{2} f_{a / A}\left(x_{1}, Q^{2}\right) f_{b / A}\left(x_{2}, Q^{2}\right) \\
\frac{d \sigma^{a b \rightarrow c d}}{d \hat{t}} \int d \epsilon P(\epsilon, \bar{\epsilon}) \frac{z_{c}^{*}}{z_{c}} \frac{D_{\pi^{0} / c}\left(z_{c}^{*}, Q^{2}\right)}{\pi z_{c}}
\end{gathered}
$$

where $z_{c}^{*}=p_{T \pi} / p_{T c}^{f}=z_{c} /(1-\epsilon)$. The parameter $\bar{\epsilon}$ determines the magnitude of the quenching and is expected decrease as the local density $\propto d N_{g} / d y$ decreases at lower energies. The $\bar{\epsilon}$ is interpreted as the average $p_{T}$ shift.

In [2] and [8], $P(\epsilon)$ was computed assuming Poisson fluctuations of the radiated gluon number. This distribution is thus characterized by the mean number of radiated gluons, $\left\langle N_{g}\right\rangle$, as well as the mean energy loss $\bar{\epsilon}$. An inherent problem of treating fluctuations via a Poisson process is that it leads to violations of the energy bound $\epsilon=\sum_{n=1}^{N_{g}} \epsilon_{n}<1$. For small $\bar{\epsilon}_{g}<0.5$ this is a small effect that can be approximately corrected by truncating the distribution at $\epsilon=1$. However, for high opacity QGP matter produced at RHIC, the gluon jet $\bar{\epsilon}_{g} \sim 0.6-0.8$ approaches unity $[8]$ and the truncation scheme leads to an approximately uniform distribution limiting $\bar{\epsilon}_{g}<0.5$.

The truncated Poisson ansatz for fluctuations provides a natural way to impose unitary and kinematic constraints on the induced radiative energy loss. However, it is possible that other distributions could be operative in the opaque limit. In this paper we explore two simplified forms of fluctuation distributions to assess some of the the systematic uncertainties in the predicted quenching factors. One is a simplified "uniform" model that reproduces the essential features of the truncated Poisson ansatz of [2, 8]. The second distribution is called "squeezed" because it accumulates strength near $\epsilon \approx 1$ in the opaque limit.

The "uniform" distribution is assumed to have the form

$$
P(\epsilon, \bar{\epsilon})= \begin{cases}\frac{\theta(0<\epsilon<2 \bar{\epsilon})}{2 \bar{\epsilon}} & \text { if } 0<\bar{\epsilon}<0.5 \\ 1 & \text { if } 0.5<\bar{\epsilon}\end{cases}
$$




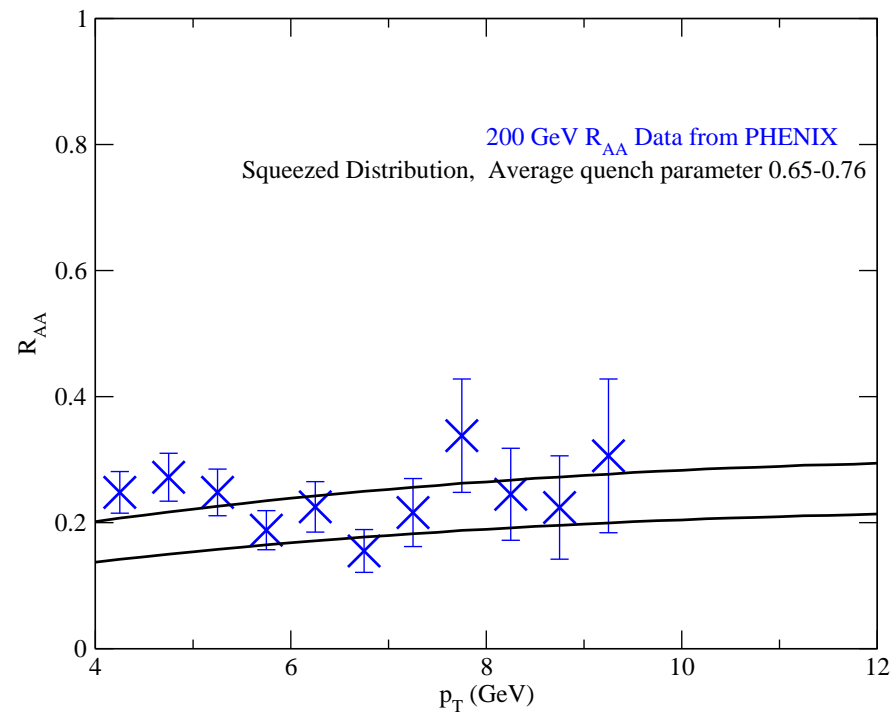

FIG. 2: $R_{A A}\left(p_{T}, \sqrt{s}=200\right)$ with squeezed fluctuations 3 The $\pi^{0}$ data are from PHENIX [4]

The "squeezed" distribution is assumed to have the form

$$
P(\epsilon, \bar{\epsilon})= \begin{cases}\frac{\theta(0<\epsilon<2 \bar{\epsilon})}{2 \overline{2} \bar{\epsilon}} & \text { if } 0<\bar{\epsilon}<0.5 \\ \frac{\theta(2 \bar{\epsilon}-\epsilon<1)}{2(1-\bar{\epsilon})} & \text { if } 0.5<\bar{\epsilon}\end{cases}
$$

Multiple gluon convolutions lead to phase space suppression $\epsilon^{n}$ of low total energy loss, which is mimicked in (3) by a simple $\theta$ function. A more detailed analysis of branching versus truncated Poisson distributions will be presented elsewhere 19]. For our present purposes of gaining a first measure of systematic errors the above overly simplified forms are adequate. Note that while the uniform distribution has a limiting $\bar{\epsilon} \leq \frac{1}{2}$ even for gluons, the "squeezed" one can easily accomodate the more realistic $\bar{\epsilon}_{g}>0.5$ situation at RHIC.

The shape of $P(\epsilon, \bar{\epsilon})$ depends not only on $\bar{\epsilon}$ but also on $\left.<N_{g}\right\rangle$, the average number of total gluons emitted. A smaller value of $\left\langle N_{g}\right\rangle$ weights the lower $n$ values more and leads to a more uniform $P(\epsilon, \bar{\epsilon})$ while larger values of $\left\langle N_{g}\right\rangle$ weight higher $n$ values [19]. The average number of gluons radiated increased with $\sqrt{s}$. Thus, we expect the uniform distribution to be a better approximation for $P(\epsilon, \bar{\epsilon})$ at $62.4 \mathrm{GeV}$ while the "squeezed" distribution could be more realistic at $200 \mathrm{GeV}$.

The nuclear modification factor can then be calculated for either distribution from Eq. 2 via

$$
R_{A A}\left(p_{T}, s\right)=\frac{d \sigma_{\pi^{0}}(\bar{\epsilon}(s))}{d \sigma_{\pi^{0}}(0)}
$$

\section{A. $R_{A A}$ with Squeezed Fluctuations}

Our first step is to fit $\bar{\epsilon}(200)$ to the $200 \mathrm{AGeV}$ PHENIX data 44 in the region $p_{T} \sim 8 \mathrm{GeV}$. Figure 2 shows the resulting $R_{A A}$. The quenched pQCD prediction for lower

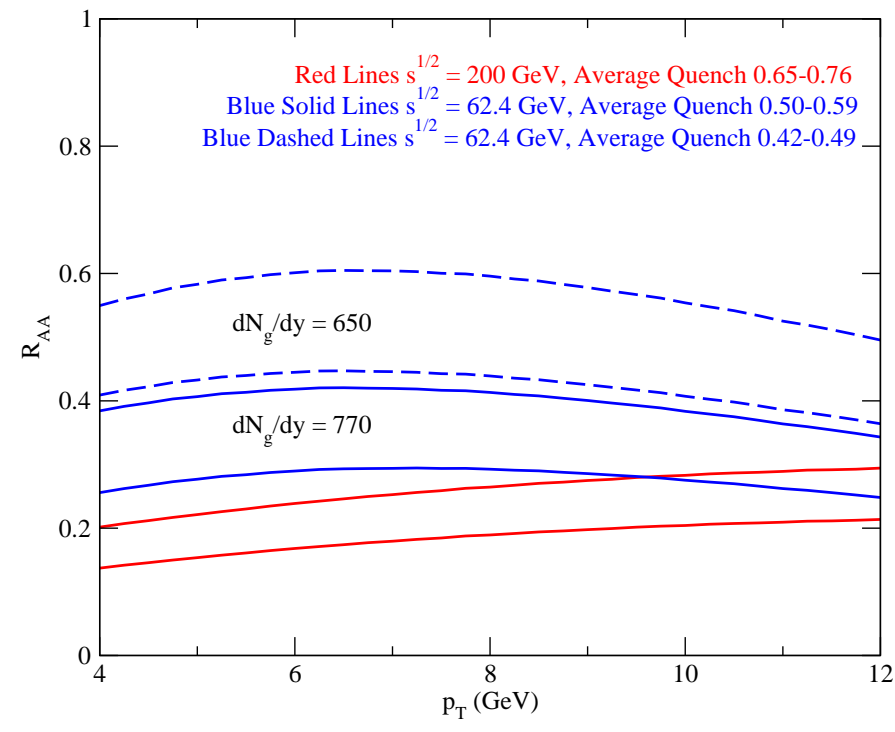

FIG. 3: Predicted energy evolution of $R_{A A}$ with squeezed fluctuations at $\sqrt{s}=62.4,200 \mathrm{GeV}$.

$p_{T}<6 \mathrm{GeV}$ increasingly deviate from the data because intrinsic $k_{T}$ smearing and Cronin calculations are neglected here. However, the approximate $p_{T}$ independence of higher $p_{T}$ is well accounted for as in [3]. The band corresponds to an assumed average mean energy loss fraction for gluons at $200 \mathrm{AGeV} 0.65<\bar{\epsilon}_{g}(200)<0.76$ using the squeezed distribution. Further below we will contrast the results to the energy evolution with the uniform distribution.

The beam energy dependence of $\bar{\epsilon}(s)$ is predicted to follow the energy dependence of the produced $d N_{g} / d y$. We also take into account the Casimir difference between quark and gluon energy loss. The mean energy loss fraction of parton type $c=q, g$ evolves with energy as

$$
\bar{\epsilon}_{c}(\sqrt{s})=\frac{C_{c}}{C_{g}}\left(\frac{d N_{g}(\sqrt{s}) / d y}{d N_{g}(200) / d y}\right) \bar{\epsilon}_{g}(200)
$$

Thus $\bar{\epsilon}_{q}=4 / 9 \bar{\epsilon}_{g}$

The predicted $R_{A A}$ at $62 \mathrm{AGeV}$ is obtained using the multiplicity systematics from PHOBOS 14 that suggests a value for $d N_{t o t} d y \approx 650-770$. From entropy conservation we expect that the gluon rapidity density is comparably decrease from about 1000 at $200 \mathrm{AGeV}$. Therefore, we expect that $\bar{\epsilon}_{g}(62.4) \approx 0.65-0.77 \bar{\epsilon}_{g}(200)$. The bands of $R_{A A}$ predictions at $62.4 \mathrm{GeV}$ are shown in Figure 3 The ranges of average quenching shown in the figure are $0.42<\bar{\epsilon}(62.4)<0.49$ and $0.50<\bar{\epsilon}(62.4)<0.59$ for multiplicity values of 650 and 770 respectively. These ranges are calculated by realizing that $\frac{d N_{g}}{d y}(200) \approx 1000$.

\section{B. $R_{A A}$ with Uniform Fluctuations}

We now repeat the $R_{A A}$ calculations in the previous section using the uniform distribution for $P(\epsilon, \bar{\epsilon})$ from 


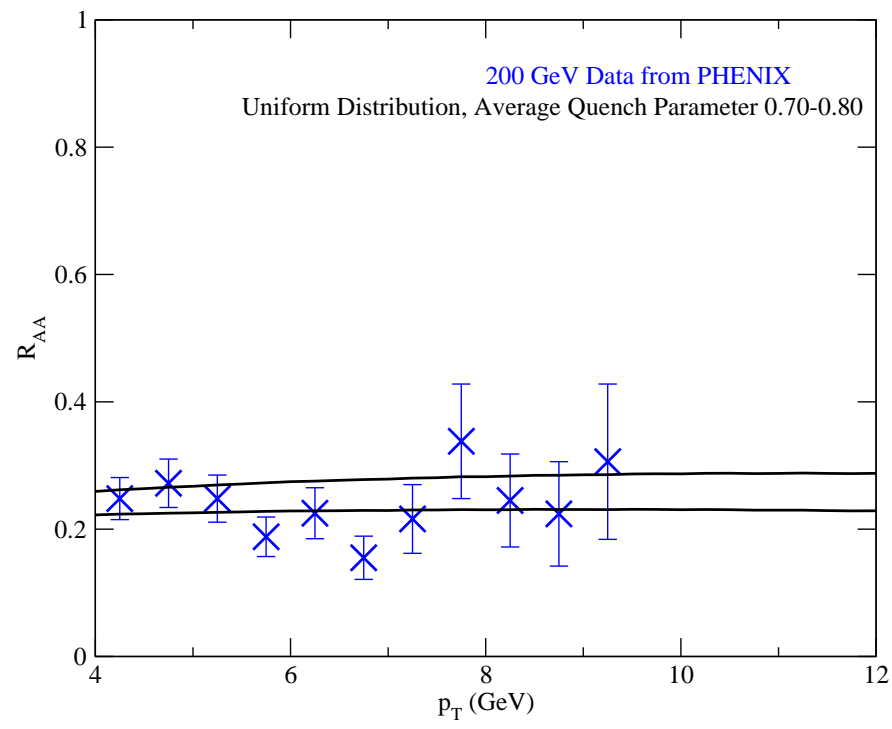

FIG. 4: $R_{A A}$ at $200 \mathrm{GeV}$ calculated using the uniform distribution. The $\pi^{0}$ data are from PHENIX [4].

Eq.(3). This is done to investigate the sensitivity of the results to the form of this distribution. The uniform distribution is the same as the squeezed distribution for $\bar{\epsilon}_{c}<0.5$. Therefore the quarks energy loss fraction distribution is identical for both distributions. However, since the gluon GLV energy loss fraction $\bar{\epsilon}_{g}>0.5$ in this energy range, the uniform distribution actually saturates the mean energy loss fraction of gluons at $\bar{\epsilon}_{g}=1 / 2$. This contrasts with the squeezed distribution that squeezes the gluon fraction energy loss toward the $\epsilon=1$ boundary. Therefore the gluon quenching is reduced with the uniform distribution while preserving the quark quenching. As we note further below, this also changes the glue to quark contribution ratios at high $p_{T}$.

$R_{A A}$ is compared to PHENIX data using the uniform fluctuation distribution in Figure 4. The curves are distinctly flatter than the ones in the previous section (Figure 21. This is because the uniform quenching distribution does not suppress so much the gluon jets than the squeezed distribution. The band in this case corresponds to $0.70<\bar{\epsilon}_{g}(200)<0.80$. In the uniform case this implies that the mean quark energy loss fraction at $\sqrt{s}=200$ is $0.31<\bar{\epsilon}_{q}(200)<0.36$

Figure 5 shows the $R_{A A}$ prediction for $62 \mathrm{AGeV}$ in this case. The range of mean gluon energy fractional energy loss is $0.46<\bar{\epsilon}_{g}(62)<0.52$ and $0.54<\bar{\epsilon}_{g}(62)<0.62$ for the different estimates of the $d N_{g} / d y(62)=650,770$ at $62 \mathrm{AGeV}$ respectively. The nuclear modification factor has a weaker energy dependence in this case and is generally somewhat smaller relative to the squeezed fluctuation case discussed in the previous section. This is due to the approximate saturation property of $\bar{\epsilon}_{g} \leq 1 / 2$, as in the full Poisson fluctuation case [8].

These calculations for the nuclear modification factor at $\sqrt{s}=62.4 \mathrm{GeV}$ are consistent with the calculations

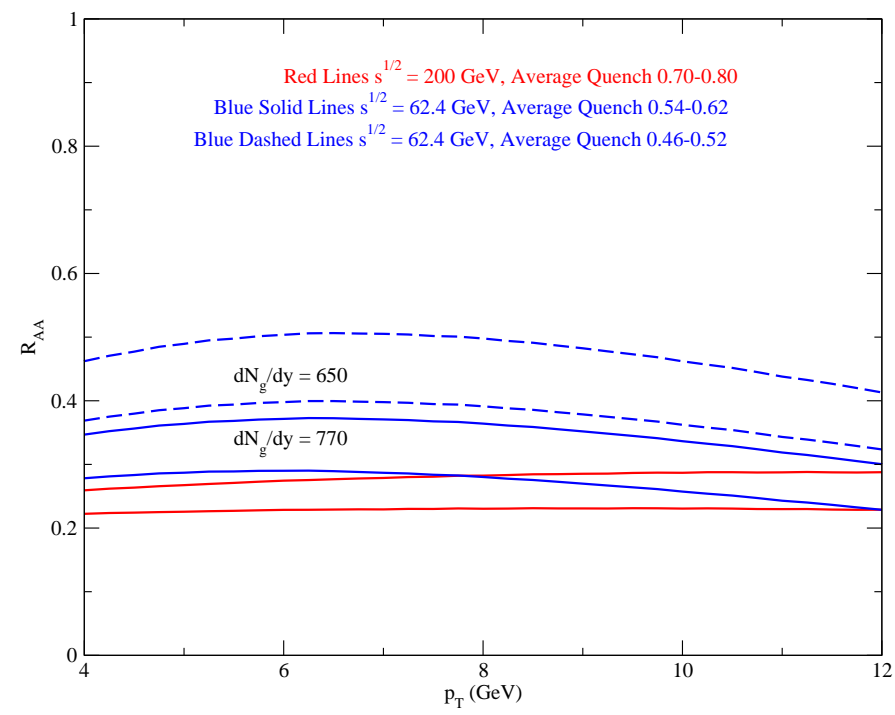

FIG. 5: $R_{A A}$ at $\sqrt{s}=62.4,200 \mathrm{GeV}$ calculated using the uniform distribution.

performed in [8]. In the $p_{T}$ region $4-6 \mathrm{GeV}$ the prediction bands of both the squeezed and uniform distributions are within the approximate range as predicted by Vitev in [8]. The main differences in the predictions can be explained by noting that the calculation in [8] includes models for Cronin interactions as well as initial parton $k_{T}$ smearing which are not included in the current calculation. Both of these effects tend to deplete hadrons in the higher transverse momentum regions and increase their number in the intermediate transverse momentum region from 2-4 GeV. This will explain the lower quenching at lower $p_{T}$ predicted by Vitev as compared to the current calculation.

Further comparisons can be attempted with recent data from the PHOBOS collaboration as seen in [6]. This publication, however, provides data at lower levels of $p_{T}$ and measures unidentified charged hadron cross-sections. This leads to a double fold problem with the comparison. Firstly, our calculation does not apply in the kinematic region probed by PHOBOS in [6]. Lower values of $p_{T}$ are not modeled well by our calculation due to previously mentioned reasons of neglecting Cronin effect and $k_{T}$ smearing. Also, the calculation of charged hadrons would only be similar to neutral pions where there were not many baryons present. The kinematic region probed by PHOBOS is dominated by baryonic data which is not taken into account in the calculation. This effect would increase $R_{A A}$ as can be seen from the data shown in $[6]$.

\section{COMPARISON OF $R_{A A}$ FOR DIFFERENT VALUES OF $\sqrt{s}$}

Note that the predicted $R_{A A}\left(p_{T}, \sqrt{s}=62\right)$ have a negative $p_{T}$ slope compared to the generally flat $R_{A A}$ at 200 $\mathrm{AGeV}$. At very high $p_{T}$, the $62.4 \mathrm{GeV}$ curves even dip be- 
low the ones at $200 \mathrm{GeV}$. This higher slope can be more robustly seen by calculating the variable $R_{s}(s)$ as

$$
R_{s}(s)=\frac{R_{A A}(s)}{R_{A A}(200)}
$$

showed in Figure 6] The figure shows multiple curves for both types of quench distributions. One can see that the $R_{s}$ curves have a distinct downward slope due to the increasing power law index of the underlying pQCD parton distributions at lower energies. These indices vary much more with $p_{T}$ at $62 \mathrm{AGeV}$ than at $200 \mathrm{AGeV}$. Therefore, the downward slope with increasing $p_{T}$ is due to the increased slope of the input spectra with increasing $p_{T}$ at lower $\sqrt{s}$.

It is remarkable that even with a smaller average energy loss at $62 \mathrm{AGeV}$, the more rapid decrease of high $p_{T}$ parton production compensates to make it appear that there is greater quenching. The $\sqrt{s}$ systematic variation of the calibrated initial jet source transverse spectra used leads to this anti-intuitive feature and must be carefully taken into account in future tomographic inversion of single and di-jet quaenching. Note that $R_{s}$ much less sensitive to the propagation of the uncertainty in $R_{A A}$ at $200 \mathrm{AGeV}$ because absolute $d N_{g} / d y$ uncertainty tends to cancel out in this $R_{s}$ ratio. This variable is also seen to be able to differentiate clearly between these two types of fluctuation distributions.

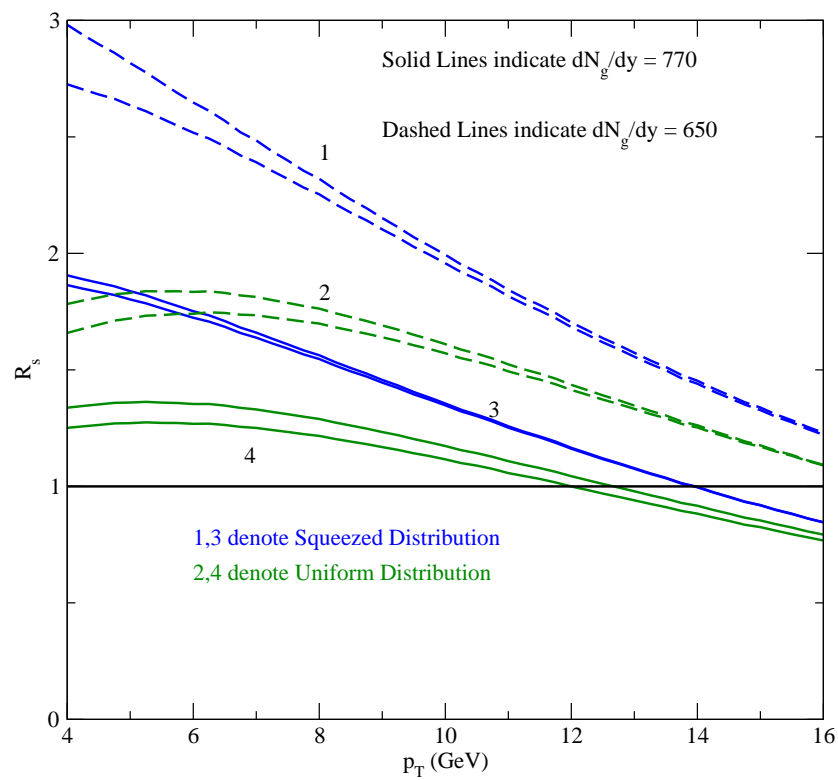

FIG. 6: The ratio of $R_{A A}$ at $62.4 \mathrm{GeV}$ to the one at $200 \mathrm{GeV}$ for different multiplicity assumptions. Curves for squeezed distributions tend to be higher than curves for uniform distributions.

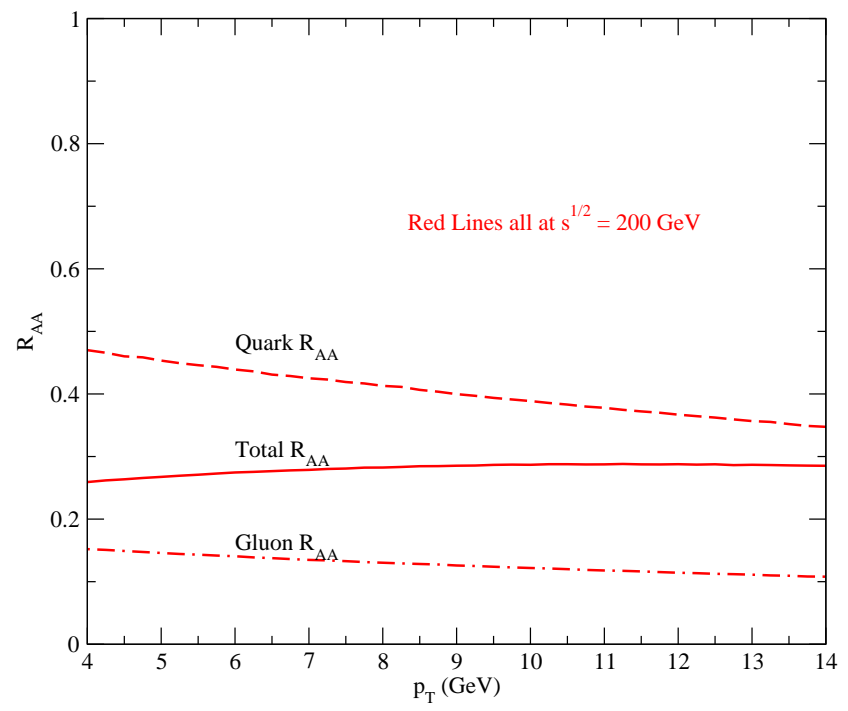

FIG. 7: The gluon and quark contributions to the total $R_{A A}^{\pi^{0}}\left(p_{T}, \sqrt{s}=200\right)$ are shown using a uniform distribution.

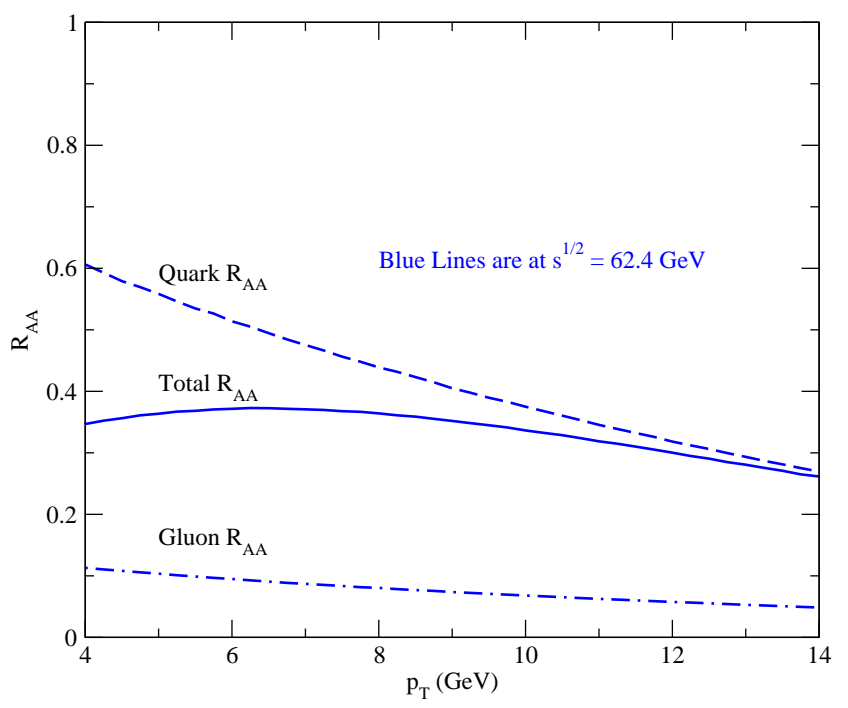

FIG. 8: The gluon and quark contributions to the total $R_{A A}^{\pi^{0}}\left(p_{T}, \sqrt{s}=62\right)$ are shown. In contrast to $\sqrt{s}=200 \mathrm{AGeV}$ the $\pi^{0}$ at $62 \mathrm{AGeV}$ are dominated by quark fragmentation.

\section{QUARK VS GLUON FRAGMENTATION}

An interesting theoretical measure of the underlying dynamics is shown in fig. (7/8)

We see that while quenched quark and gluon fragmentation contributes comparable amounts to the final quenched $\pi^{0}$ spectrum at $200 \mathrm{AGeV}$, The gluon contribution is almost completely quenched by $62 \mathrm{AGeV}$ in our calculations in spite of the smaller $\bar{\epsilon}_{g}$ at 62 . The quark and gluon $p_{T}$ transverse evolve with $s$ in different calculable ways in $\mathrm{pQCD}$. This evolution however causes 


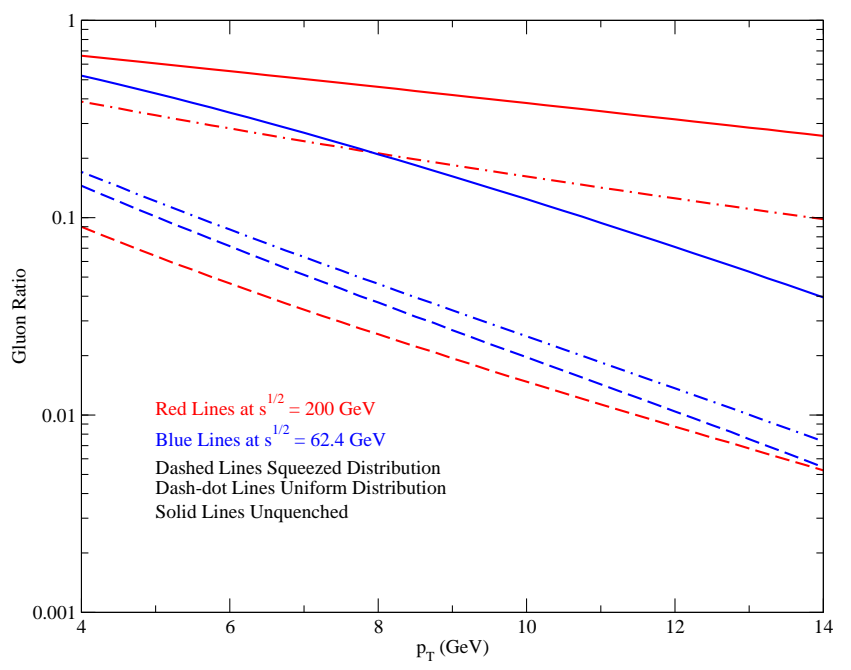

FIG. 9: The ratio of $\pi^{0}$ from gluons both quenched and unquenched at $\sqrt{s}=62.4,200 \mathrm{GeV} \cdot \frac{d N_{g}}{d y}(62.4)$ is assumed to 770. Lower levels of quenching are used to make this estimate. $\bar{\epsilon}_{\text {squeezed }}$ is 0.65 and 0.50 at $200 \mathrm{GeV}$ and $62.4 \mathrm{GeV}$ respectively. $\bar{\epsilon}_{\text {uniform }}$ is 0.7 and 0.54 at $200 \mathrm{GeV}$ and 62.4 $\mathrm{GeV}$ respectively.

the rapid disappearance of the gluonic contribution as $s$ decreases in the case of the uniform distribution.

In Fig 9] we see that the form of the fluctuation distribution strongly influences the pion from gluon jet fraction. For the squeezed distribution, the gluon jet contribution is already negligible at $200 \mathrm{AGeV}$ and of course remains so at 62 modulo a small increase due to the decrease of $\bar{\epsilon}(62)$ relative to 200 .
These results suggest that identified particle ratios sensitive to quark vs gluon fragmentation [16 in the high $p_{T}>5 \mathrm{GeV}$ domain would provide valuable to constrain on the form of the energy loss fluctuations and to map out more accurately the interplay of quark and gluon contributions.

\section{CONCLUSIONS}

Predictions for $R_{A A}\left(p_{T}, \sqrt{s}=62\right)$ for $\pi^{0}$ were made with a focus on the systematic uncertainties resulting from as yet un-tested assumptions about the energy loss fluctuation spectrum. The most unintuitive aspect of the prediction is a decreasing $R_{A A}$ with $p_{T}$ at lower energies that is due to the more rapid decrease of the produced parton $p_{T}$ distributions. The negative $p_{T}$ slope of $R_{A A}$ provides an important constraint on the fluctuation distributions. The pion fractions from gluon jets were also shown to be sensitive to the underlying fluctuation distributions. Identified particle ratios at $p_{T}>5 \mathrm{GeV}$ should help to further test the distribution. Such further studies are essential in order to calibrate more accurately the jet tomographic measure of the initial QGP densities produced at RHIC.

\section{Acknowledgments}

Discussions with I. Vitev and X.N. Wang are gratefully acknowledged. This work is supported in part by the United States Department of Energy under Grants No. DE-FG02-93ER40764.
[1] M. Gyulassy, I. Vitev, X. N. Wang and B. W. Zhang, arXiv:nucl-th/0302077

[2] M. Gyulassy, P. Levai and I. Vitev, Phys. Lett. B 538, 282 (2002) arXiv:nucl-th/0112071.

[3] I. Vitev and M. Gyulassy, Phys. Rev. Lett. 89, 252301 (2002).

[4] S. S. Adler et al. [PHENIX Collaboration], Phys. Rev. Lett. 91, 072301 (2003) arXiv:nucl-ex/0304022; Phys. Rev. Lett. 91, 241803 (2003) arXiv:hep-ex/0304038; Phys. Rev. C 69, 034910 (2004) arXiv:nucl-ex/0308006.

[5] J. Adams et al. [STAR Collaboration], Phys. Rev. Lett. 91, 172302 (2003) arXiv:nucl-ex/0305015.

[6] B. B. Back [the PHOBOS Collaboration], arXiv:nucl-ex/0405003

[7] X. N. Wang, Phys. Lett. B 579, 299 (2004) arXiv:nucl-th/0307036.

[8] I. Vitev, arXiv:nucl-th/0404052

[9] I. Vitev and M. Gyulassy, Phys. Rev. C 65, 041902 (2002) arXiv:nucl-th/0104066.

[10] T. Hirano and Y. Nara, Phys. Rev. C 68, 064902 (2003).

[11] R. J. Fries, B. Muller, C. Nonaka and S. A. Bass, Phys.
Rev. Lett. 90, 202303 (2003) arXiv:nucl-th/0301087. D. Molnar and S. A. Voloshin, Phys. Rev. Lett. 91, 092301 (2003) arXiv:nucl-th/0302014.

[12] M. Gyulassy, I. Vitev and X. N. Wang, Phys. Rev. Lett. 86, 2537 (2001) arXiv:nucl-th/0012092.

[13] R. Baier, Y. L. Dokshitzer, A. H. Mueller and D. Schiff, JHEP 0109, 033 (2001) arXiv:hep-ph/0106347.

[14] B. B. Back et al. [PHOBOS Collaboration], Phys. Rev. Lett. 88, 022302 (2002) arXiv:nucl-ex/0108009.

[15] U. A. Wiedemann and C. A. Salgado, Phys. Rev. D68, 014008(2003). arXiv:hep-th/0302184.

[16] G. I. Fai, G. G. Barnafoldi, M. Gyulassy, P. Levai, G. Papp, I. Vitev and Y. Zhang, arXiv:hep-ph/0111211

[17] A. D. Martin, W. J. Stirling and R. G. Roberts, Phys. Lett. B 306, 145 (1993). For a more recent global analysis see A. D. Martin, W. J. Stirling, R. G. Roberts and R. S. Thorne, Eur. Phys. J. C 4, 463 (1998)

[18] J. Binnewies,B. A. Kniehl and G. Kramer, Z. Phys. C 65, 471 (1995).

[19] A. Adil and M. Gyulassy, in preparation. 